\title{
Optimizing image guidance frequency and implications on margins for gynecologic malignancies
}

\author{
Carmen Stromberger ${ }^{1 *}$, Arne Gruen ${ }^{1}$, Waldemar Wlodarczyk', Volker Budach', Christhardt Koehler ${ }^{2}$
} and Simone Marnitz ${ }^{1}$

\begin{abstract}
Background: To analyze setup deviations using daily megavoltage computed tomography (MVCT) and to evaluate three MVCT frequency reducing protocols for gynecologic cancer patients treated with helical tomotherapy.

Methods: We recorded the setup errors of 56 patients with gynecological cancer observed throughout their whole course by matching their daily MVCT with the planning CT. Systematic and random errors were calculated on a patient and population basis. We defined three different protocols corresponding to MVCTs from the first five fractions (FFF), the first ten fractions (FTF) or from the first and third weeks (505). We compared theoretical. setup errors calculated using these 5 or 10 early MVCT scans with the actual errors found with the remaining fractions to to analyze the residual deviations.
\end{abstract}

Results: The total systematic (random) deviations had means of $-2.0(3.8) \mathrm{mm}, 0.5(3.4) \mathrm{mm}, 0.5(6.1) \mathrm{mm}$ and $-0.5^{\circ}$ $\left(0.9^{\circ}\right)$ in vertical $(V)$, longitudinal $(L O)$, lateral $(L A)$, and roll $(R)$ directions, respectively. The proposed three MVCT protocols resulted in minor residual deviations. In all three protocols, $95 \%$ of all calculated residual deviations were less than or equal to $5 \mathrm{~mm}$ in all 3 directions. When examining the additional minimal CTV-PTV setup margins that were calculated based on these residual deviations, the 505 protocol would have allowed smaller margins than the FFF and FTF protocol, particularly in the $V$ direction.

Conclusions: For patients with gynecologic cancer, the 505-protocol led to the lowest residual deviations and therefore might offer the best approach in reducing the frequency of pre-treatment MVCTs.

Keywords: Helical tomotherapy, MVCT, Setup accuracy, Gynecological tumors, Image guidance, Margin

\section{Background}

The daily use of image guidance (IGRT) is finding more frequent application in radiation therapy. Modern linear and helical tomotherapy (HT) accelerators are equipped with an onboard imaging device e.g. $\mathrm{kV}$ (kilovoltage; cone-beam) CT scanner or MVCT scanner (megavoltage) to localize the target and are capable of applying intensity modulated radiotherapy (IMRT) or volumetric modulated arc therapy (VMAT) to deliver highly conformal dose distributions [1]. HT uses an MVCT imaging tool [2] and has been established for the treatment

\footnotetext{
* Correspondence: carmen.stromberger@charite.de

'Department of Radiooncology, Charité Universitätsmedizin Berlin, Campus

Virchow Klinikum, Augustenburger Platz 1, Berlin 13353, Germany

Full list of author information is available at the end of the article
}

of patients with gynecologic malignancies [3,4]. For a curative radiotherapy or chemoradiation an adequate treatment of the CTV is essential. The CTV to PTV margin is an established planning method to enable appropriate coverage of the target volume. Pelvic radiotherapy is associated with both an overall higher setup uncertainty in patient positioning and uncertainties due to potential internal organ motion (e.g. uterus, rectum, prostate), thus necessitating wide PTV margins. The shrinkage of the CTV-PTV margin might lead to a reduction of treatment related toxicity (e.g. gastrointestinal) but too narrow margins will increase the risk of inadequate cancer treatment, especially for techniques with highly conformal doses to the target volume. The MVCT of HT allows a daily patient setup verification and correction prior to each 
treatment, but the scan acquisition, matching with the $\mathrm{KV}$-planning $\mathrm{CT}$ and patient repositioning takes significant additional time, depending on the image scan length. This has an impact on patient comfort and tolerance. In this study we investigated the feasibility of reducing the frequency of MVCT scans for patients with gynecological tumors by determining patient and population based systematic and random errors. Three different reduced frequency protocols were analyzed with regard to their respective residual deviations were they to be used in the daily routine.

\section{Methods}

\section{Patients and patient setup}

We analyzed data from a total of 56 patients with cervical cancer, FIGO stage IB-IVA, treated with HT at our institute from June 2008 to December 2009. Pelvic radiotherapy was delivered in 48 cases, 8 patients were treated with pelvic and extended field radiotherapy because of paraaortic lymph node metastases. A total of 34 patients were treated with definitive chemoradiation, 22 patients were treated with an adjuvant radio(chemo)therapy. All patients were placed in a supine position and immobilized using a combiboard with knee-ankle fixation (Unger Medizintechnik, Germany) with their arms folded over their chest. Planning CT scans (CT scanner LightSpeed ${ }^{\circledR}$ from GE Healthcare, General Electric Company, NYSE; GE) were performed at a slice thickness of $3.75 \mathrm{~mm}$.

CTV definition, PTV margins, organ at risks, dose prescription and planning parameters for definitive chemoradiation with or without paraaortic (extended) field irradiation have been described earlier [3]. For postoperative treatment, the CTV included all regions of potential microscopic disease: the surgical bed, regional lymph node areas (common, external and internal iliacs and the presacral region), and the vaginal cuff. The planning target volume (PTV) was outlined as the CTV plus $1 \mathrm{~cm}$ in all directions. The caudal field border was at the obturator foramen, the upper field border was individualized on the basis of the patient's anatomy to include the common iliac lymph nodes. Patients were initially positioned by aligning skin surface markings with the treatment room's lasers.

\section{Treatment verification}

MVCTs were typically acquired before each fraction allowing a daily patient setup verification and correction. The scan region and length were defined by the radiation oncologist on the first day of treatment and used for all future scans. The PTV was not routinely scanned over its whole length for patients with a paraaortic extended field. In general, MVCTs were acquired in the normal mode (slice thickness of $4 \mathrm{~mm}$ ), with a scan length between $15-20 \mathrm{~cm}$. The resulting images were visualized at the HT workstation and the system's software was used to automatically determine the setup accuracy. A fine resolution matrix $(256 \times 256)$ and a variation of mutual information registration called extracted feature fusion based on a mixed "bony and tissue" anatomy were used to rigidly co-register the MVCT images with those from the planning CT, a feature offered by the HT software. Deviations were calculated in the lateral (LA; $x$ axis), longitudinal (LO; $y$ axis), vertical ( $\mathrm{V} ; \mathrm{z}$ axis) direction and for rotation in the roll ( $R$; in y axis). Pitch and yaw rotational deviations had to be kept at $0^{\circ}$, since these could not be corrected for by couch or gantry manipulations. Deviations in pitch and yaw rotation were corrected by manually patient repositioning and controlled with a repeat MVCT scan. The initial automatic correction was verified by a radiation oncologist and any necessary corrections were made using bony structures, the soft tissue of the cervical region/tumor (definitive treatment) and PTV localization. Following the registration procedure, the total deviations were corrected for by a shift of the treatment couch in the LO, V and LA directions. The couch used from September 2008 until June 2009 had to be manually adjusted in the LA direction, but was later replaced by a fully automatic couch. Rotational, roll (R) angle was corrected by the fully computerized alteration of the linear accelerator. Radiotherapy was delivered in a mean of 28 fractions (range: 27-31), amounting to a total of 1564 MVCTs.

\section{Calculation of errors and analysis of imaging protocols}

The calculation of the systematic and random setup errors was performed according to the different parameters published by others $[5,6]$.

The total deviation data in the LO, LA, V, and R directions, acquired by daily MVCTs prior to treatment were retrospectively evaluated. These results were used to generate and compare different protocols for the imaging frequencies. The first five fractions (FFF) protocol is calculated on the basis of the patients' MVCTs acquired on the first five days of radiotherapy. The averages of the LO, LA and $\mathrm{V}$ deviations (average total deviation per direction) were calculated and theoretically applied for the subsequent treatments (23 fractions). This protocol resembles the FFF-protocol with five image fractions previously described by Vaandering and colleagues, though this was used for the treatment of the head and neck and brain region [7]. The first ten fractions (FTF) protocol is based and calculated on the patients' MVCTs acquired during the first ten fractions (days 1-10). The averages of the detected LO, LA and V deviations (average total deviation per direction) were calculated and theoretically applied to the subsequent treatments (18 fractions). The alternate week (505) protocol was based on the 5 MVCTs on days 1-5 and the 5 MVCT scans on days 11-15. The scans for fractions 6-10 were not used 
for the calculation. The LO, LA and V deviations were then averaged and theoretically applied to the subsequent 13 fractions. For all three protocols, the differences in setup errors in all four degrees of freedom (residual deviations) were calculated by subtracting the averaged total deviations calculated under the specific protocol from the actual deviations detected for the remaining fractions. These residual deviations were then used to determine cumulative distribution functions $(\mathrm{CDF})$. The CDF is a function describing the probability that deviations no greater than the associated deviation (random variable of the $\mathrm{CDF}$ ) will occur. The steeper the CDF, the smaller the residual deviation and the better the MVCT frequency protocol. The CDFs were determined using built-in functions of Microsoft Excel and used to compare the three different MVCT protocols. Furthermore, we calculated the smallest CTV-PTV margin that is needed if these protocols were applied according to Van Herk's formula and McKenzie's IMRT assumption: 2.5 Rres (systematic deviation) +0.7 rres (random deviation) ensuring that $90 \%$ of the patients receive a minimum dose of $95 \%$ to the CTV $[5,6,8]$.

\section{Statistical analysis}

Microsoft Excel 2007 and IBM SPSS Version 20 were utilized to calculate the data and to obtain descriptive statistics of the patients' deviations. Student's two-sided $\mathrm{t}$-tests were calculated using a significance level of 0.05 .

\section{Results}

The systematic and random components of all setup deviations (LO, LA and $\mathrm{V}$ directions) were calculated allowing the analysis of the mean systematic deviation $(\mathrm{M})$, the standard deviation of the systematic error $(\Sigma)$ and the mean random error $(\sigma)$ for all patients (Table 1 , Figure 1). Deviations in roll direction were small $\left(\Sigma=0.7^{\circ}\right.$, $\left.\mathrm{M}=-0.5^{\circ}, \sigma=0.9^{\circ}\right)$. We did see a negative shift of the patients' systematic deviations in the $\mathrm{V}$ direction due to a known upward pitch of the HT couch. This has been described elsewhere as a $3 \mathrm{~mm}$ maximum shift depending on the longitudinal position of the couch [7].

Residual deviations resulting from the application of the three imaging frequency protocols are shown in Figure 2. The 505 protocol resulted in significantly

Table 1 Systematic and random deviations

\begin{tabular}{|c|c|c|c|}
\hline & $\begin{array}{c}\text { Systematic } \\
\text { deviation (M) }\end{array}$ & $\begin{array}{l}\text { Standard deviation of } \\
\text { all systematic deviations }(\Sigma)\end{array}$ & $\begin{array}{l}\text { Mean of all random } \\
\text { deviations }(\sigma)\end{array}$ \\
\hline LO & $0.5 \mathrm{~mm}$ & $3.7 \mathrm{~mm}$ & $3.4 \mathrm{~mm}$ \\
\hline V & $-2.0 \mathrm{~mm}$ & $5.7 \mathrm{~mm}$ & $3.8 \mathrm{~mm}$ \\
\hline LA & $0.5 \mathrm{~mm}$ & $4.0 \mathrm{~mm}$ & $6.1 \mathrm{~mm}$ \\
\hline
\end{tabular}

Total systematic and random deviations prior to correction for gynecologic patients $(n=56)$ in the LO, V and LA directions. Shown are the populations' mean of all systematic deviations (M), the standards deviations of all systematic deviations $(\Sigma)$ and the mean of all random deviations $(\sigma)$. smaller residual deviations in the $\mathrm{V}$ and $\mathrm{LA}$ direction compared to the FFF $(\mathrm{p}<0.001)$ and FTF $(\mathrm{p}<0.02)$ protocol. No significant difference could be seen in the LO direction. In the FFF and FTF protocol, $5 \%$ of the calculated residual vertical deviations were larger than $3 \mathrm{~mm}$ compared to $2 \%$ for the 505 protocol. In the FFF protocol, $95 \%$ of the calculated LO residual deviations were smaller than $4 \mathrm{~mm}$ compared to $3 \mathrm{~mm}$ for the FTF or 505 protocol, respectively. The residual LA deviations in $95 \%$ of the FFF and FTF protocol, were calculated to be smaller or equal to $5 \mathrm{~mm}$ compared to $4 \mathrm{~mm}$ for the 505 protocol. In all three protocols, $95 \%$ of all calculated residual deviations were less than or equal to $5 \mathrm{~mm}$ in all 3 directions. When examining the additional minimal CTV-PTV setup margins that were calculated using these residual deviations, the 505 protocol resulted in smaller margins than the FFF and FTF protocol, particularly in the $\mathrm{V}$ direction (Table 2).

\section{Discussion}

For patients with gynecologic tumors, HT, conventional IMRT and proton radiotherapy allow normal organ sparing and highly conformal doses to the target volume, with or without an additional simultaneous integrated boost to high-risk areas (e.g. parametria) [3,4,9,10]. An accurate target delineation, highly reproducible patient immobilization, and a clear understanding of internal-organ motion and tumor shrinkage are prerequisites for the optimal use of these sophisticated techniques on such patients [11-13]. The margin definition has a great impact on DVH parameters for the organs at risk in pelvic radiotherapy, especially the small bowel $[10,14]$. Wide range of different CTV-PTV margins in the treatment of gynecologic malignancies has been published in the literature [15-17]. Up to now, there is no generally accepted standard. Furthermore, to the best of our knowledge, there is not yet sufficient data available to judge the true usefulness of frequent (daily) image guided setup correction $[15,18-20]$ and any impact on margin size or imaging protocols aimed at reducing the frequency of daily imaging for patients with gynecologic malignancies. Techniques and acute toxicities of such treatment have been reported previously [3,9]. A significant inter-fractional ( $>3$ days) improvement of setup accuracy in the $\mathrm{V}$ direction (not for LO and LA) has been reported, reflecting a learning curve over time (patient-, technician-wise) has been reported [18]. The use of 3D-imaging over the first few days has been suggested [19].

In order to support - or vary - our institutional routine of using daily MVCTs and a $1 \mathrm{~cm}$ CTV to PTV margin in all directions, we retrospective determined the daily setup deviations with respect to three different imaging frequency protocols for 56 gynecologic cancer patients and analyzed their systematic and random components. Our data also suggest a learning curve for patients 

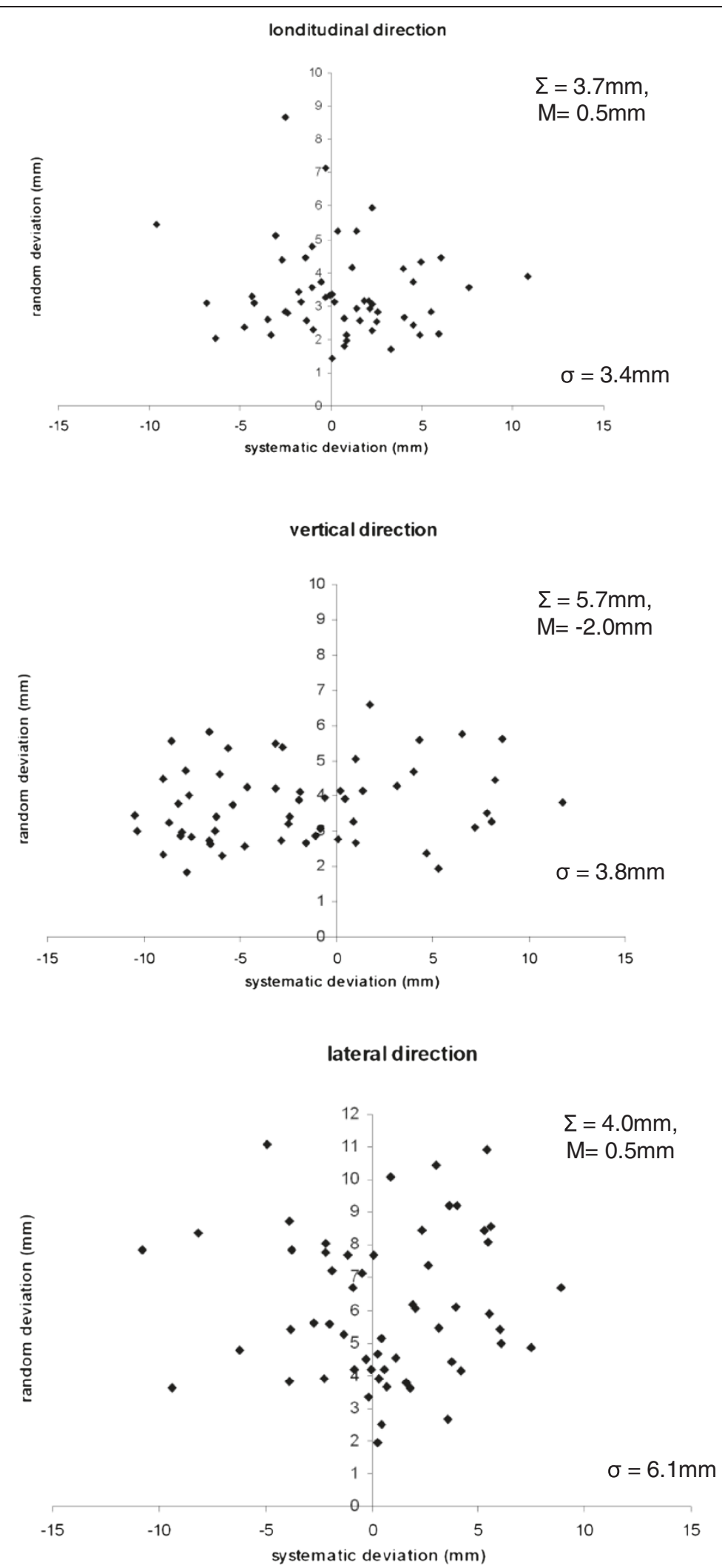

Figure 1 Total systematic and random deviations for gynecologic patients $(n=56)$ in the LO, V and LA directions. Figures include the populations' mean of all systematic deviations $(M)$, the standards deviations of all systematic deviations $(\Sigma)$ and the mean of all random deviations $(\sigma)$. 

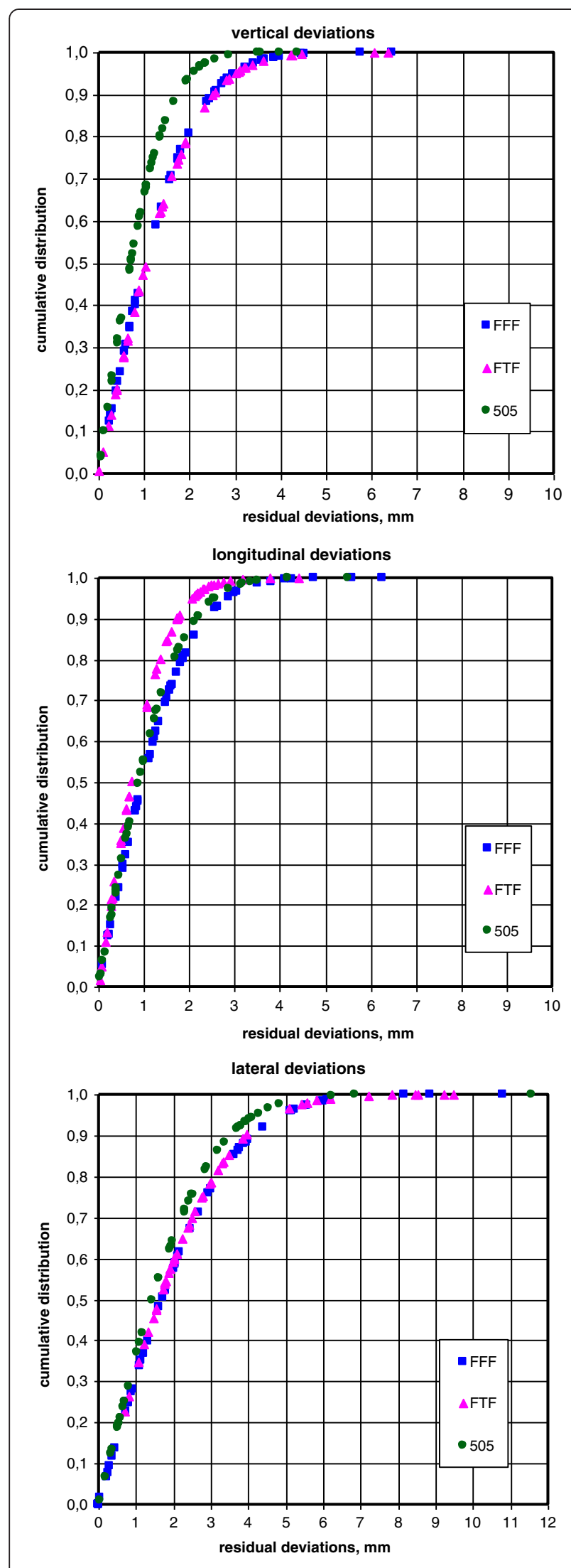

Figure 2 Residual deviations of MVCT protocols. Cumulative distribution of the residual deviations in $\mathrm{V}, \mathrm{LA}$ and $\mathrm{LO}$ directions persisting when applying the FFF (blue rectangle), the FTF (pink triangle), the 505 protocol (green dot) for gynecologic tumor patients. $p<0.05$ in $V$ and LA for the 505 protocol.

and/or technicians, since not only the FTF was superior to the FFF protocol but the 505 led to the best result, where indeed the images of fraction 10-15 in addition to the first 5 were used for the calculation of our model. Data based on additional post-treatment MVCT-images seems to indicate that patient motion contributes less than internal organ motion to the whole setup error $(1.1 \pm 1.3 \mathrm{~mm}$ anterior-posterior, $-0.3 \pm 1.6 \mathrm{~mm}$ lateral, $0.2 \pm 2.3 \mathrm{~mm}$ cranio-caudal) than internal organ motion does [17].

Although there is inconclusive data on uterus/cervix organ motion in the literature, patients setup errors might be reduced to a minimum via daily imaging and corrections [15]. The decrease in setup error as proposed by any of our imaging protocols might help to reduce margins. In all our protocols, $95 \%$ of all calculated residual deviations were less than or equal to $5 \mathrm{~mm}$ in all 3 directions. Our results showed that the application of the 505 protocol can lead to smaller $(<1 \mathrm{~cm})$ CTV-PTV margins at least in areas where organ motion is limited (e.g. vessels/lymph nodes) for definitive and also potentially for postoperative HT, IMRT or VMAT treatment. Not only inter-fractional setup errors but also the intra-fractional movements (organ motion), inter-fractional anatomical changes (bladder- and rectum filling, small bowel movement), potential changes in BMI and tumor shrinkage need to be accounted for. Nevertheless, the uterus/cervical motion, changes in BMI and tumor shrinkage have not been part of our study. However, a study conducted by Collen and colleagues [17] investigated the cervical organ motion in 10 patients with daily MVCTs and found deviations of $-3.5 \pm 4.9 \mathrm{~mm}$ to the left, $0.2 \pm 4.5 \mathrm{~mm}$ to the right, $0.5 \pm 10.1 \mathrm{~mm}$ in the anterior, $-3 \pm 6.9 \mathrm{~mm}$ in the

Table 2 CTV-PTV margins

\begin{tabular}{ccccc}
\hline & \multicolumn{4}{c}{ CTV-PTV margin } \\
\cline { 2 - 5 } & \multicolumn{3}{c}{$\begin{array}{c}(\mathbf{2 . 5} \times \text { Rres (systematic deviation) } \\
\text { deviation due to organ }\end{array}$} \\
\cline { 2 - 5 } & $\mathbf{v}$ (mm) & lo $\mathbf{~ ( m m ) ~}$ & la $(\mathbf{m m})$ & $\mathbf{r}\left(^{\circ}\right)$ \\
\hline Uncorrected & 14.9 & 10.2 & 11.6 & 1.9 \\
FFF & 8.4 & 8.8 & 13.4 & 1.7 \\
FTF & 7.0 & 6.6 & 11.5 & 1.7 \\
$\mathbf{5 0 5}$ & 5.5 & 6.0 & 9.2 & 1.4 \\
\hline
\end{tabular}

The calculated minimal CTV-PTV set-up margins for the vertical (V), longitudinal (LO), lateral (LA) and angular (R) deviations for three different MVCT frequency protocols (FFF: first five fractions; FTF: first ten fractions; 505: on alternate weeks: 1st and 3rd week MVCTs). 
posterior, $2.2 \pm 8.0 \mathrm{~mm}$ in the superior and $0.5 \pm 5 \mathrm{~mm}$ in the inferior direction, respectively. Uterus motion seems to be even more pronounced than cervical movement $[16,17]$. Taylor et al. found uterus deviations of $2.7 \pm 2.8 \mathrm{~mm}$ anterior-posterior, $4.1 \pm 4.4 \mathrm{~mm}$ superior-inferior and lateral of $0.3 \pm 0.8 \mathrm{~mm}$ using MR-imaging on two consecutive days and they recommended margins of $15 \mathrm{~mm}$ (anteriorposterior), $15 \mathrm{~mm}$ (superior-inferior), and $7 \mathrm{~mm}$ lateral for the nodal regions and the parametria [16]. Kaatee et al. [21] found a large range of cervical organ movements using a fluoroscopic electronic portal imaging device and radioopaque markers. The sigma shifts of the markers in the anterior-posterior, superior-inferior, and lateral direction were 3.5, 4.3 and $3.4 \mathrm{~mm}$, respectively. These findings lead them to recommend margins of 10.5, 12.2 and $9.2 \mathrm{~mm}$. Van de Bunt and colleagues [22] used MR-images prior to and after IMRT treatment for cervical cancer patients. The variations in organ movement they found led to propose much larger CTV-PTV margins of $24 \mathrm{~mm}$ (anterior), $17 \mathrm{~mm}$ (posterior), $11 \mathrm{~mm}$ (superior), $8 \mathrm{~mm}$ (inferior), $12 \mathrm{~mm}$ (right) and $16 \mathrm{~mm}$ (left). Santanam et al. [15] recommend a $7 \mathrm{~mm}$ CTV-PTV margins in all directions when using daily imaging and daily setup corrections. Stroom et al. [23] proposed a $5 \mathrm{~mm}$ CTV-PTV margin based on a study on 14 patients with user defined landmarks $(\mathrm{kV}, \mathrm{MV}$ orthogonal Portal imaging). Although a CTV-PTV margin of 20/10 $\mathrm{mm}$ was used in the study, Lim and colleagues [24] could show that a $5 \mathrm{~mm}$ margin might be appropriate for most patients treated with IMRT with the use of a small bowel displacement system if daily setup control is used. The uterus fundus was not part of the CTV in this study. Furthermore, conflicting data exist on the impact of organ filling (bladder, rectum) on cervical movement. Some authors found a correlation between rectum volume/filling and cervical organ motion [16] and/or no correlation with bladder filling $[16,25]$ others observed no correlation of bladder or rectum filling with cervical organ motion [22].

To evaluate the possibility of reducing patients' "time on machine" by using protocols that allow less frequent imaging but maintaining the higher accuracy offered by IGRT, a further study is required to look into patient comfort via quality of life questionnaires. The practical effect of our protocols on CTV-PTV margins and delivered dose distributions need to be further analyzed.

\section{Conclusions}

This study retrospectively analyzed patient setup deviations for gynecologic cancer patients. Daily positioning and setup correction have improved treatment setup accuracy. We investigated the feasibility of reducing the frequency of pretreatment MVCTs by calculating the residual deviations that would arise when applying three different imaging frequency protocols. The 505 protocol resulted in significantly smaller residual deviations particularly in the $\mathrm{V}$ and LA directions, but not in the LO direction. An analysis of its viability, the global impact on cutting down treatment time and therefore improvements patient comfort are needed to provide more conclusive evidence for a benefit in treatment of gynecologic patients.

\section{Consent}

Written informed consent was obtained from the patient for publication of this report and any accompanying images.

\section{Competing interests}

There are no actual or potential conflicts of interest.

\section{Authors' contributions}

CS carried out the studies, participated in the design, analyzed and interpreted the data and drafted the manuscript. WW participated in the design of the study, performed the statistical analysis and interpreted the data. AG, VB, CK, SM conceived of the study, and participated in its design and coordination and helped to draft the manuscript. All authors read and approved the final manuscript.

\section{Author details}

'Department of Radiooncology, Charité Universitätsmedizin Berlin, Campus Virchow Klinikum, Augustenburger Platz 1, Berlin 13353, Germany. ${ }^{2}$ Department of Gynecology, Charité Universitätsmedizin Berlin, Berlin, Germany.

Received: 8 March 2013 Accepted: 19 April 2013

Published: 2 May 2013

\section{References}

1. Forrest L, Mackie TR, Ruchala K, Turek M, Kapatoes J, Jaradat H, Hui S, Balog J, Vail DM, Mehta MP: The utility of megavoltage computed tomography images from a helical tomotherapy system for setup verification purposes. Int J Radiat Oncol Biol Phys 2004, 60:1639-1644.

2. Mackie TR, Kapatoes J, Ruchala K, Lu W, Wu C, Olivera G, Forrest L, Tome W, Welsh J, Jeraj $R$, et al: Image guidance for precise conformal radiotherapy. Int J Radiat Oncol Biol Phys 2003, 56:89-105.

3. Marnitz S, Stromberger C, Kawgan-Kagan M, Wlodarczyk W, Jahn U, Schneider A, Ulrich U, Budach V, Kohler C: Helical tomotherapy in cervical cancer patients: simultaneous integrated boost concept: technique and acute toxicity. Strahlenther Onkol 2010, 186:572-579.

4. Marnitz S, Lukarski D, Kohler C, Wlodarczyk W, Ebert A, Budach V, Schneider A, Stromberger C: Helical tomotherapy versus conventional intensitymodulated radiation therapy for primary chemoradiation in cervical cancer patients: an intraindividual comparison. Int I Radiat Oncol Biol Phys 2011, 81:424-430

5. McKenzie AL, van Herk M, Mijnheer B: The width of margins in radiotherapy treatment plans. Phys Med Biol 2000, 45:3331-3342.

6. van Herk M: Errors and margins in radiotherapy. Semin Radiat Oncol 2004, 14:52-64

7. Vaandering A, Lee JA, Renard L, Gregoire V: Evaluation of MVCT protocols for brain and head and neck tumor patients treated with helical tomotherapy. Radiother Oncol 2009, 93:50-56.

8. van Herk M, Remeijer P, Rasch C, Lebesque JV: The probability of correct target dosage: dose-population histograms for deriving treatment margins in radiotherapy. Int J Radiat Oncol Biol Phys 2000, 47:1121-1135.

9. Marnitz S, Kohler C, Burova E, Wlodarczyk W, Jahn U, Grun A, Budach V, Stromberger C: Helical tomotherapy with simultaneous integrated boost after laparoscopic staging in patients with cervical cancer: analysis of feasibility and early toxicity. Int I Radiat Oncol Biol Phys 2012, 82:e137-e143.

10. Georg D, Georg P, Hillbrand M, Potter R, Mock U: Assessment of improved organ at risk sparing for advanced cervix carcinoma utilizing precision radiotherapy techniques. Strahlenther Onkol 2008, 184:586-591.

11. Chen MF, Tseng CJ, Tseng CC, Kuo YC, Yu CY, Chen WC: Clinical outcome in posthysterectomy cervical cancer patients treated with concurrent 
Cisplatin and intensity-modulated pelvic radiotherapy: comparison with conventional radiotherapy. Int J Radiat Oncol Biol Phys 2007, 67:1438-1444.

12. Kidd EA, Siegel BA, Dehdashti F, Rader JS, Mutic S, Mutch DG, Powell MA, Grigsby PW: Clinical outcomes of definitive intensity-modulated radiation therapy with fluorodeoxyglucose-positron emission tomography simulation in patients with locally advanced cervical cancer. Int I Radiat Oncol Biol Phys 2010, 77:1085-1091.

13. Mundt AJ, Roeske JC, Lujan AE, Yamada SD, Waggoner SE, Fleming G, Rotmensch J: Initial clinical experience with intensity-modulated wholepelvis radiation therapy in women with gynecologic malignancies. Gynecol Oncol 2001, 82:456-463.

14. Ahamad A, D'Souza W, Salehpour M, lyer R, Tucker SL, Jhingran A, Eifel PJ: Intensity-modulated radiation therapy after hysterectomy: comparison with conventional treatment and sensitivity of the normal-tissue-sparing effect to margin size. Int J Radiat Oncol Biol Phys 2005, 62:1117-1124.

15. Santanam L, Esthappan J, Mutic S, Klein EE, Goddu SM, Chaudhari S, Wahab S, El Naqa IM, Low DA, Grigsby PW: Estimation of setup uncertainty using planar and MVCT imaging for gynecologic malignancies. Int J Radiat Oncol Biol Phys 2008, 71:1511-1517.

16. Taylor A, Powell ME: An assessment of interfractional uterine and cervical motion: implications for radiotherapy target volume definition in gynaecological cancer. Radiother Oncol 2008, 88:250-257.

17. Collen C, Engels B, Duchateau M, Tournel K, De Ridder M, Bral S, Verellen D, Storme G: Volumetric imaging by megavoltage computed tomography for assessment of internal organ motion during radiotherapy for cervical cancer. Int J Radiat Oncol Biol Phys 2010, 77:1590-1595.

18. Hui SK, Lusczek E, DeFor T, Dusenbery K, Levitt S: Three-dimensional patient setup errors at different treatment sites measured by the Tomotherapy megavoltage CT. Strahlenther Onkol 2012, 188:346-352.

19. Schubert LK, Westerly DC, Tome WA, Mehta MP, Soisson ET, Mackie TR, Ritter MA, Khuntia D, Harari PM, Paliwal BR: A comprehensive assessment by tumor site of patient setup using daily MVCT imaging from more than 3,800 helical tomotherapy treatments. Int J Radiat Oncol Biol Phys 2009, 73:1260-1269.

20. Zeidan OA, Huddleston AJ, Lee C, Langen KM, Kupelian PA, Meeks SL, Manon RR: A comparison of soft-tissue implanted markers and bony anatomy alignments for image-guided treatments of head-and-neck cancers. Int J Radiat Oncol Biol Phys 2010, 76:767-774.

21. Kaatee RS, Olofsen MJ, Verstraate MB, Quint S, Heijmen BJ: Detection of organ movement in cervix cancer patients using a fluoroscopic electronic portal imaging device and radiopaque markers. Int I Radiat Oncol Biol Phys 2002, 54:576-583.

22. van de Bunt $L$, Jurgenliemk-Schulz IM, de Kort GA, Roesink JM, Tersteeg RJ, van der Heide UA: Motion and deformation of the target volumes during IMRT for cervical cancer: what margins do we need? Radiother Oncol 2008, 88:233-240.

23. Stroom JC, Olofsen-van Acht MJ, Quint S, Seven M, de Hoog M, Creutzberg $\mathrm{CL}$, de Boer HC, Visser HC: On-line set-up corrections during radiotherapy of patients with gynecologic tumors. Int J Radiat Oncol Biol Phys 2000, 46:499-506.

24. Lim K, Kelly V, Stewart J, Xie J, Cho YB, Moseley J, Brock K, Fyles A, Lundin A, Rehbinder $\mathrm{H}$, Milosevic M: Pelvic radiotherapy for cancer of the cervix: is what you plan actually what you deliver? Int I Radiat Oncol Biol Phys 2009, 74:304-312.

25. Kerkhof EM, Raaymakers BW, van der Heide UA, van de Bunt L, JurgenliemkSchulz IM, Lagendijk JJ: Online MRI guidance for healthy tissue sparing in patients with cervical cancer: an IMRT planning study. Radiother Oncol 2008, 88:241-249.

doi:10.1186/1748-717X-8-110

Cite this article as: Stromberger et al:: Optimizing image guidance frequency and implications on margins for gynecologic malignancies. Radiation Oncology 2013 8:110.

\section{Submit your next manuscript to BioMed Central and take full advantage of:}

- Convenient online submission

- Thorough peer review

- No space constraints or color figure charges

- Immediate publication on acceptance

- Inclusion in PubMed, CAS, Scopus and Google Scholar

- Research which is freely available for redistribution

Submit your manuscript at www.biomedcentral.com/submit
Biomed Central 